\section{MS33-P2 Crystal structure determination of two novel}

\section{1,2,4-triazolo[3,4-b]-1,3,4-thiadiazine and -thiadiazole derivatives by XRPD method}

Filiz B. Kaynak ${ }^{1}$, Gülsüm Gündoğdu ${ }^{1}$, Melanie Müller ${ }^{2}$, Süheyla Özbey ${ }^{1}$

1. Hacettepe University, Faculty of Engineering, Department of Physics Engineering, Beytepe, 06800, Ankara, TURKEY

2. Ruhr University Bochum Institute for Geology Mineralogy and Geophysics Crystal Chemistry, 44721, Bochum, GERMANY

email: gulsen@hacettepe.edu.tr

In recent years, studies reporting anticancer activities of a number of fused 1,2,4-triazole derivatives such as triazolopyridazine, triazolotriazine, triazolothiadiazine have being attracting interest. In the light of these knowledge, we prepared new series of 1,2,4-triazolo[3,4- $b]$-1,3,4-thiadiazine and 1,2,4-triazolo[3,4- $b]-1,3,4$ - thiadiazole derivatives and evaluated their possible anticancer activities [1]. The samples are originally synthesized in Hacettepe University, Faculty of Pharmacy, Department of Pharmaceutical Chemistry by Dr. Tozkoparan and her group.

In the presented research, we have obtained and determined crystal structures of two compounds by X-ray powder diffraction techniques. The synchrotron measurements of the samples have been done in Argonne National Laboratory on 11-BM beamline. Data sets were collected over the $1-40^{\circ} 2 \theta$ range with a $0.001^{\circ}$ step size at room temperature $(295.0 \mathrm{~K})$ with $(\lambda \sim 0.459169 \AA)$ wavelength. This measurement from beamline 11-BM at APS allowed us to fully analyze this data.

The powder X-ray diffraction pattern of the samples were first indexed using the TOPAS 4.2 program. Structure determination of compound I was carried out using the simulated annealing method implemented in the program DASH (unit cell of compound 1: $\mathrm{a}=15.55645(11) \AA, \mathrm{b}=8.61693(6) \AA, \mathrm{c}=8.56702(6) \AA$, $\beta\left({ }^{\circ}\right)=104.3270(4)$, space group: $\mathrm{P} 2$ ) and FOX program was used for compound II (unit cell of compound 2: $\mathrm{a}=6.373(3) \quad \AA, \quad \mathrm{b}=11.362(5) \quad \AA, \quad \mathrm{c}=14.089(6) \AA$, $\alpha\left(^{\circ}\right)=80.175(1), \beta\left(^{\circ}\right)=94.877(1), \gamma\left(\left(^{\circ}\right)=99.022(1)\right.$, space group: P-1). XRD patterns are carefully analyzed by the Rietveld method using FullProf program and soft restraints was employed at the final stage of the refinement. Good agreement was achieved between calculated and experimental powder diffraction patterns at the final Rietveld refinement (Figure 1). So, the agreement factors support the correctness of the structures (for compound 1: $R_{w p}=0.0694 R_{p}=0.05470$ $\mathrm{R}_{\text {exp }}=0.0704$ and $\left.\mathrm{Chi}^{2}=0.9705\right)$.

Financial support to Gülsüm Gündoğdu of the Scientific and Technical Research Council of Turkey is gratefully acknowledged (grants 2214 A by TÜBITAK one year research grant during $\mathrm{PhD}$ ). We also thank Argonne National Laboratory for the synchrotron measurement of compound I and II using 11-BM mail-in Program.

[1] P. S. Aytaç, I. Durmaz, D. R. Houston, R.Ç.-Atalay, B. Tozkoparan, Bioorganic \& Medicinal Chemistry, Vol 24, Iss 4, 2016, 858-872.

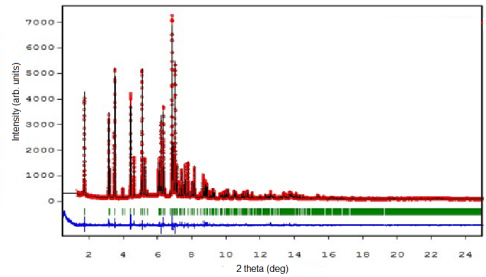

Figure 1. FullProf Rietveld refinement solution for compound 1.

Keywords: XRPD, Rietveld refinement, 1,2,4-triazole derivatives 